\title{
CCD observations of Phoebe, 9th satellite of Saturn
}

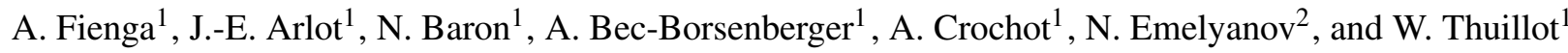 \\ 1 Institut de mécanique céleste et de calcul des éphémérides, Observatoire de Paris, EP 1825 CNRS, \\ 77 avenue Denfert-Rochereau, 75014 Paris, France \\ 2 Sternberg Institute, Moscow, Russia and Institut de mécanique céleste et de calcul des éphémérides, Observatoire de Paris, \\ France
}

Received 29 March 2002 / Accepted 24 May 2002

\begin{abstract}
In 1998 and 1999, we started observations of the 9th satellite of Saturn. We made 163 observations using the $120 \mathrm{~cm}-$ telescope of Observatoire de Haute-Provence, France. We used the USNO A2 catalogue of stars for the astrometric reduction. With the help of observations of optical counterparts of ICRF sources, a zonal correction to the USNO A2.0 catalogue was computed and applied to the Phoebe positions. A comparison with the most recent theories was made.
\end{abstract}

Key words. planets and satellites: general - astrometry

\section{Introduction}

The theoretical model and the ephemerides of the motion of the 9th satellite of Phoebe need an improvement in their accuracy because of the lack of high-accuracy observations referred directly to modern star catalogues. We started a campaign of observation in order to provide this improvement. This paper provides the data with explanations on the reduction performed. These data can also be accessed on the data base NSDC dedicated to the natural satellites (http://www. bdl.fr/nsdc.html) $)^{1}$.

\section{The campaign of observations}

\subsection{The existing sets of observations}

Phoebe, the 9th satellite of Saturn was discovered in 1898 by Pickering. Several series of photographic observations were made in order to allow the making of ephemerides. The motion of Phoebe is not fast: the period of revolution is $\mathbf{5 4 6}$ days and 14 hours. The inclination is of 174 degrees, which means the motion is retrograde around the planet compared to the other satellites. However, the quality of the ephemerides decreases if no observations are made for several years. The distribution of the observations made since the discovery are plotted in Fig. 1. The number of observations versus the year are presented. We added our observations obtained during the 1998 1999 campaign.

\section{Send offprint requests to: A. Fienga, e-mail: fienga@bdl . fr}

1 These data are also available at the CDS via anonymous ftp to cdsarc.u-strasbg.fr (130.79.128.5) or via

http://cdsweb.u-strasbg.fr/cgi-bin/qcat?J/A+A/391/767

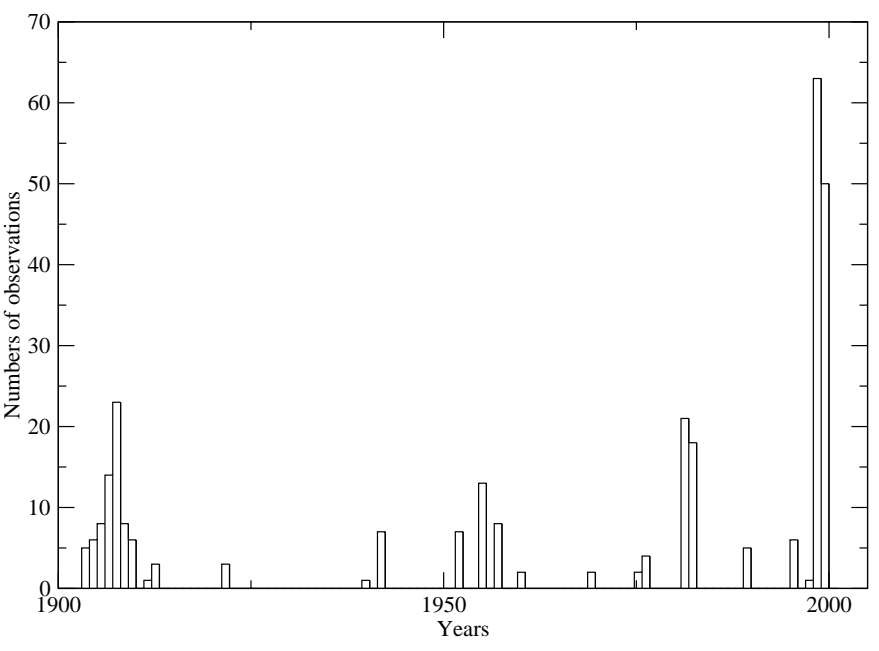

Fig. 1. Distribution of the observations made from 1898 to 2000.

\subsection{The observations made}

The observations were made at Observatoire de HauteProvence, France, using the $120 \mathrm{~cm}$ telescope. The focal length is about $7 \mathrm{~m}$. The CCD has a $1024 \times 1024$ pixels target with a scale of 0.68 arsec per pixel. The field of the target is about $12 \mathrm{~min}$ of a degree.

84 images were made in August 1998, 10 in September 1998, 46 images were made in December 1998, 16 images were made in October 1999 and 7 images in November 1999. Figure 2 shows the distribution of the data along the orbit of Phoebe during our campaign of observations. One can see that the observations cover about half an orbit. Furthermore, the size of the field of view (about 12 min by 


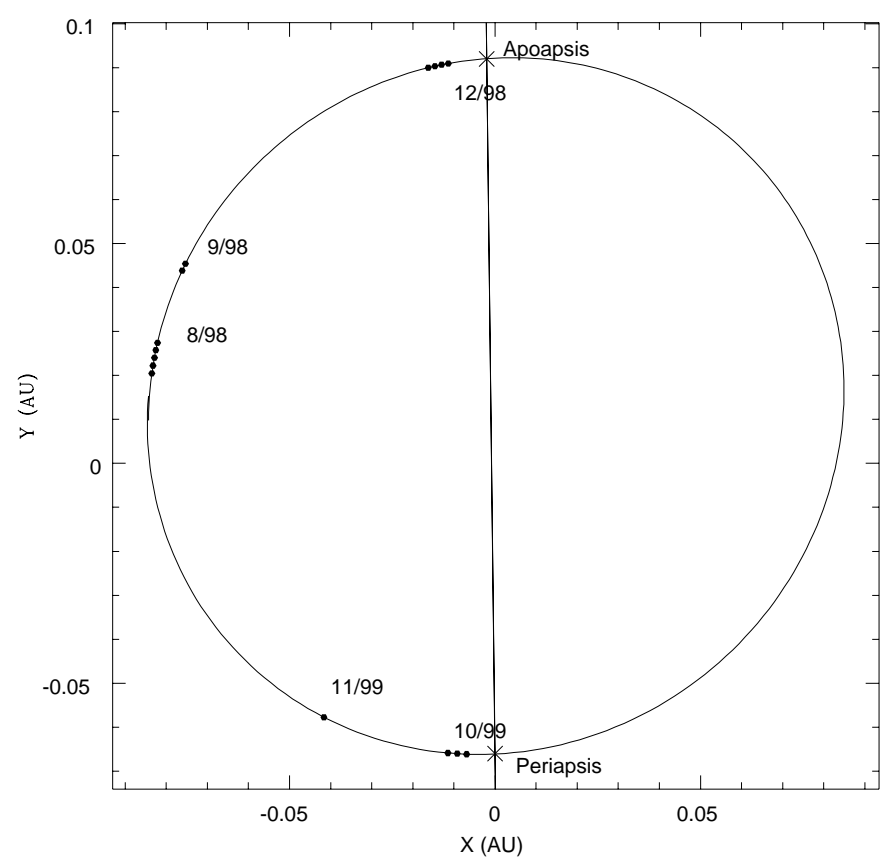

Fig. 2. Positions of the observations of Phoebe on its orbit around Saturn. The marked positions were observed from December 1998 to November 1999 in 5 sessions (see Table 1).

12 min of square degree) is large enough to make a direct reduction of the image.

\subsection{Available theories}

Two numerical theories of Phoebe's motion are currently used. The first one (Jacobson 1998) is mainly based on groundbased and Voyager observations. The second one (Arlot et al. 2002) is based only on groundbased observations but is fitted to recent observations realized by Veiga et al. (2000) from 1995 to 1997 , by Stone (2000), and Stone \& Harris (2000) from 1995 to 2000. In this paper, we will compare the observed positions with both of these theories. As it will appear in the next paragraphs, no large differences are contributed by the choice of the theories.

\section{The astrometric reduction}

\subsection{The measurements and the calibration of the field: Determination of absolute positions}

The calibration of the field (determination of the constants of the target in order to determine the positions of Phoebe) was made using the USNO A2 catalogue (Monet et al. 1998) because of the large number of stars available easily covering the field of $12 \times 12 \mathrm{~min}$ of a degree.

All the images reduced in this paper were treated with two original software sets developped at the Institut de Mécanique Céleste (Berthier et al. 2000). The first software set, called CCDPHOT and based on MIDAS routines (Banse et al. 991), deals with the treatment and the calibration of the CCD images, the automatic detection and measurements of objects, and the connection to stellar catalogues. The second sotware set, called
Table 1. Means and standard deviations, in arcseconds, of the observed minus the computed right ascension and declination of Phoebe.

\begin{tabular}{rrr}
\hline \hline$(\mathrm{O}-\mathrm{C})$ & Jacobson $(1998)$ & Arlot et al. (2002) \\
\hline$\Delta \alpha \cos (\delta)\left[{ }^{\prime \prime}\right]$ & $(0.156 \pm 0.148)$ & $(0.167 \pm 0.162)$ \\
$\Delta \delta\left[{ }^{\prime \prime}\right]$ & $(0.465 \pm 0.177)$ & $(0.470 \pm 0.187)$ \\
\hline
\end{tabular}

PRIAM (Fortran 90 library) provides a very accurate process of astrometric reduction (Fienga 1998; Fienga et al. 2002).

The flatfielding and the subtraction of the background noise is performed using the MIDAS tools. The extraction of the sources is made using a Gaussian stellar profile and with extraction of the background noise before the fit of the Gaussian profile. To perform the manual cross identification between the extracted sources and the different stellar catalogues, a pipeline was set up based on SEXTRACTOR (Bertin 1998) and the WCStools (Mink 1995). The output of the pipeline are two files: one containing the catalogue number of the objects used as references, and their positions in pixels on the CCD, the other one is the list of the stellar references with their astrometric characteristics (catalogue numbers, positions, proper motions if available...). The last file can be modified by the user or replaced as an input of PRIAM by another file built by the user.

PRIAM (Fienga 1999) performs an accurate reduction of the images. An easy-to-use interface was built on the web (http://www.bdl.fr/priam.html). The inputs of PRIAM are the two output files of the pipeline and a series of observational characteristics such as the geodetic coordinates of the observer, the meteorological parameters during the session of observations, the type of filters. The reduction is done including computations of star ephemerides in apparent coordinates (correction of annual, diurnal aberrations, deflection of light), correction of refraction by mapping functions dealing with the meteorological conditions of observations and the wavelengths of observations. These estimations are completed by comparaison with the dynamical theory of the scientific objects of interest, if this theory exists.

\section{Results and interpretation}

\subsection{Phoebe positions in the USNO A2.0 frame}

Figure 3 provides the O-C's of the set of data for two ephemerides: the one of Arlot et al. (2002) and the one of Jacobson (1998). The rms of the residuals are given in Table 2. The positions of Phoebe computed by the two models are quite close. Both of these theories of motion are numerical integrations of the same perturbations of the Phoebe orbit. The remaining differences can then be justified by the different sets of observations used to adjust the numerical integrations. More accurate descriptions of the perturbations included in the numerical integrations and the sets of observations used in the fit of the orbit are available in Arlot et al. (2002) and Jacobson (1998).

The offset of about 0.44 arcsec in declination in Fig. 3 and Table 2 is more significant. This offset could be induced by several factors: the methods of measurement and reduction, 

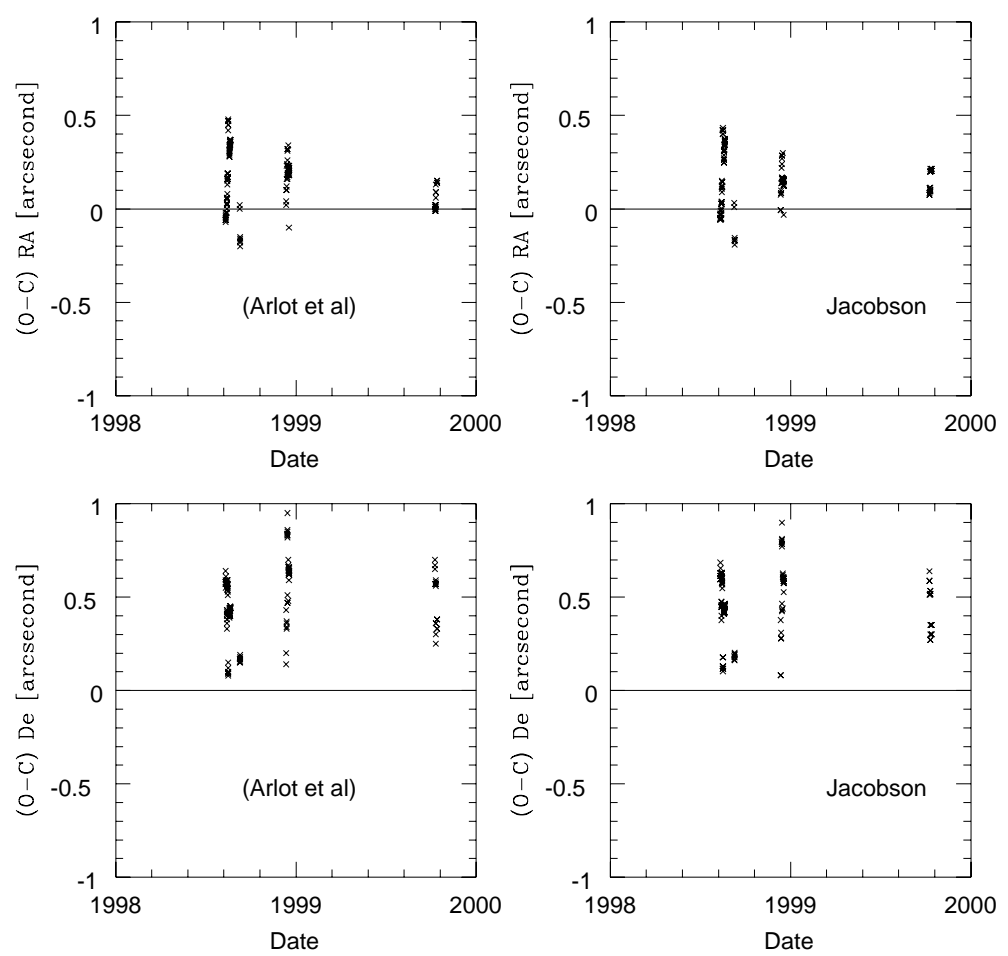

Fig. 3. Right ascension and Declination residuals in arcseconds. The comparison were made between the observed positions and the ones computed with the two available theories: Arlot et al. (2002) and Jacobson (1998).
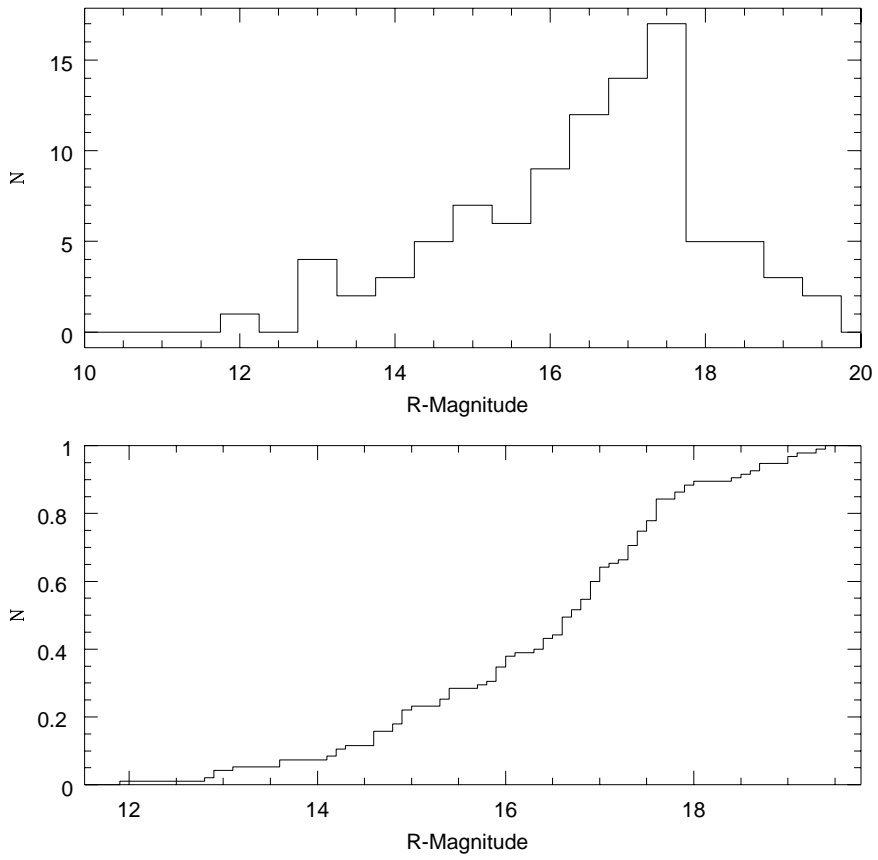

Fig. 4. Histogram and cumulative histogram of the distribution of the $R$-magnitude of the USNO2.0 stars used in the computation of the Phoebe positions. The mean of this distribution is $(16.4 \pm 1.6)$. As it appears in the cumulative histogram, all the stars are fainter than the 11th magnitude and more than $70 \%$ of the stars have a magnitude greater than 16.

a mis-modeling of the atmospheric refraction, or a zonal defect in the catalogue. The applied methods of measurement and reduction were used in several cases with complete success
(Fienga 1998; Berthier et al. 2000; Arlot et al. 2002; BonnetBidaud et al. 2000...). Studies were carried out on the impact of possible mis-modelings of the atmospheric refraction. As it was demonstrated in Berthier \& Fienga (1999), none of them could induce such an offset in a 2-year period.

Furthermore, Assafin et al. (2001) detected offsets in the USNO A2.0 star declinations by comparison between the catalogues USNO A2.0, ACT (Urban et al. 1998) and the improved GSC (Morrison et al. 1999). Mainly, a zonal error around $+30^{\circ}$ declination was detected in the USNOA2.0 for stars fainter than the 11th magnitude. The plate measurements used to set up the USNO catalogue were referred to the ACT, meaning that stars fainter than the ACT magnitude range could be affected by zonal errors. The mean magnitude of Phoebe at the opposition is 16.5 , meaning that the USNO2.0 stars used in the reduction process have a mean $R$-magnitude greater than 11 (cf. Fig. 4). Such zonal error in the USNOA2.0 catalogue described by Assafin et al. could then affect the observed positions of Phoebe, as we will see next.

\subsection{ICRF sources in the USNO A2.0 frame}

In December 2001, Fienga observed 40 ICRF sources in optical wavelengths (540 and $610 \mathrm{~nm})$ at the Observatoire de Haute-Provence, France, using the $120 \mathrm{~cm}$ telescope (Fienga $\&$ Andrei 2002). The configuration of the instrument remains unchanged since the Phoebe observations. The objects are observed from 23 hours to 13 hours in right ascension and from 0 degree to 45 degrees in declination. The visual magnitudes of the sources are fainter than the 11th magnitude as well as the magnitude of the stars used in the reduction process. 
Table 2. Means and standard deviations, in arcseconds, of the observed minus ICRF right ascension and declination of the 40 ICRF sources observed in optical wavelengths and reduced using the USNO A2.0 catalogue (Cols. 3 and 4), and the preliminary version of the GSC II catalogue (Cols. 5 and 6).

\begin{tabular}{cccccc}
\hline \hline $\begin{array}{c}\text { Declination } \\
\text { Zones }\end{array}$ & Number of ICRF sources & $\begin{array}{c}\text { USNO A2.0 } \\
\Delta \alpha \cos \delta \\
\operatorname{arcsec}\end{array}$ & $\begin{array}{c}\Delta \delta \\
\operatorname{arcsec}\end{array}$ & $\begin{array}{c}\text { GSC II } \\
\operatorname{arcsec} \delta\end{array}$ & $\begin{array}{c}\Delta \delta \\
\operatorname{arcsec}\end{array}$ \\
\hline $0^{\circ}<\delta<45^{\circ}$ & 40 & $(0.030 \pm 0.173)$ & $(0.254 \pm 0.153)$ & $(0.057 \pm 0.131)$ & $(0.059 \pm 0.138)$ \\
& & & & \\
$0^{\circ}<\delta<10^{\circ}$ & 13 & $(0.079 \pm 0.158)$ & $(0.225 \pm 0.175)$ & $(0.056 \pm 0.147)$ & $(0.075 \pm 0.165)$ \\
$10^{\circ}<\delta<20^{\circ}$ & 21 & $(0.020 \pm 0.189)$ & $(0.291 \pm 0.139)$ & $(0.058 \pm 0.137)$ & $(0.063 \pm 0.131)$ \\
$20^{\circ}<\delta<30^{\circ}$ & 3 & $(0.214 \pm 0.098)$ & $(0.158 \pm 0.013)$ & $(0.102 \pm 0.085)$ & $(-0.013 \pm 0.110)$ \\
$30^{\circ}<\delta$ & 3 & $(0.017 \pm 0.093)$ & $(0.207 \pm 0.203)$ & $(0.015 \pm 0.054)$ & $(0.035 \pm 0.115)$ \\
\hline
\end{tabular}

A detailed description of this work is given in (Fienga \& Andrei 2002). It was possible to extract from this study very interesting residuals. These residuals were obtained by comparison between ICRF positions and positions of these sources reduced with USNO A2.0 stars and stars extracted from the preliminary version of the GSC II (STScI 2001). By this method, we estimate possible offsets on ICRF source positions induced by USNO A2.0 stars and observed with the same instrument as Phoebe.

Such residuals do not represent only the offsets between the stellar catalogues. They also contain offsets between the positions of the radio sources and their optical counterparts. As demonstrated by (Charlot 1990; Fey \& Charlot 1997), the position of an extragalactic radio-source and the position of its optical counterpart can be different. This difference is induced by the structure of the source, but because of the lower precision and accuracy of our optical observations of the counterparts, this does not affect our study (Zacharias et al. 1999).

As it appears in Table 2 (Fienga \& Andrei 2002), such offsets between ICRF positions and positions obtained with USNO A2.0 stars exist.

A global offset of $(0.258 \pm 0.157)$ arcsecond can be estimated for the region $0^{\circ}<\delta<20^{\circ}$. This offset does not appear with the positions deduced from the GSC II stars, meaning that the USNO A2.0 seems to present a zonal error.

\subsection{Zonal correction}

During the 1998-1999 period, Phoebe shows variations of declination from $+8.5^{\circ}$ to $+13^{\circ}$. It is then possible to correct the Phoebe positions for the zonal error detected previously. We estimate that the zonal offsets in the $+8^{\circ}$ to $+13^{\circ}$ degrees zone are $(0.002 \pm 0.199)$ arcsec in right ascension and $(0.311 \pm 0.140)$ arcsec in declination. No significant offset was estimated in right ascension; however, corrections are applied in declination. Table 3 presents the new residuals corrected for the zonal error. No significant offset remains.

The new residuals are compared with the ones obtained by Veiga et al. (2000). The observations of Phoebe are also re-
Table 3. Means and standard deviations, in arcseconds, of the observed minus the computed right ascension and declination of Phoebe with zonal corrections deduced from ICRF sources observations.

\begin{tabular}{lcc}
\hline \hline$(\mathrm{O}-\mathrm{C})$ & Jacobson (1998) & Arlot et al. (2002) \\
with zonal correction & & \\
\hline$\Delta \alpha \cos (\delta)\left[^{\prime \prime}\right]$ & $(0.156 \pm 0.148)$ & $(0.167 \pm 0.162)$ \\
$\Delta \delta\left[{ }^{\prime \prime}\right]$ & $(0.154 \pm 0.177)$ & $(0.159 \pm 0.187)$ \\
\hline
\end{tabular}

duced using the USNO A2.0 catalogue. However, during the period observed by Veiga et al., the declination of Phoebe goes from $-04^{\circ}$ to $+05^{\circ}$, meaning that the USNO A2.0 zone used in the reduction process is not the same as the one studied in this work. In right ascension, no significant offset is detected and the dispersions are similar $(0.14$ arcsec with Veiga and 0.17 arcsec in this paper). In declination, a small but significant offset $(0.29 \pm 0.26)$ is found in Veiga et al., despite a quite important dispersion. A possible explanation of such residuals could by a bad fit of the Phoebe orbit near the periapsis (Veiga et al.). No clear evidence of confirmation of such a suggestion can be found in our results because the main part of our observations were taken away from this region.

\section{Observed positions}

Table 4 provides the set of observations. These data are available in the NSDC (Natural Satellites Data Center) database ${ }^{2}$. The data given in Table 4 are as follows: in Col. 1 are given the Dates of observation in UTC, and in Cols. 2 and 3, the topocentric ICRF J2000 right ascensions and declinations of Phoebe are given.

\footnotetext{
${ }^{2} \mathrm{ftp}: / /$ www.bdl.fr/ftp/pub/NSDC/saturn/rawdata/ positions/
} 
Table 4. Observed positions of Phoebe. These positions are topocentric, ICRF J2000 and corrected from the USNO A2.0 zonal error. Column 1 gives the Dates of Observation in UTC.

\begin{tabular}{|c|c|c|c|c|c|}
\hline $\begin{array}{c}\text { Date of Observation } \\
\text { Y M D UTC }\end{array}$ & $\begin{array}{l}\text { Right ascension } \\
\text { h m s.sss }\end{array}$ & $\begin{array}{l}\text { Declination } \\
\text { d m s.ss }\end{array}$ & $\begin{array}{c}\text { Date of Observation } \\
\text { Y M D UTC }\end{array}$ & $\begin{array}{l}\text { Right ascension } \\
\text { h m s.sss }\end{array}$ & $\begin{array}{l}\text { Declination } \\
\text { d m s.ss }\end{array}$ \\
\hline 19980812.02070870 & 021044.425 & +103015.68 & 19980816.10601870 & 021053.601 & +102959.25 \\
\hline 19980812.02397870 & 021044.437 & +103015.68 & 19980816.10928870 & 021053.606 & +102959.25 \\
\hline 19980812.02920870 & 021044.451 & +103015.64 & 19980816.11255870 & 021053.610 & +102959.21 \\
\hline 19980812.03223870 & 021044.461 & +103015.60 & 19980816.11907870 & 021053.618 & +102959.14 \\
\hline 19980812.03528870 & 021044.468 & +103015.64 & 19980816.12234870 & 021053.621 & +102959.14 \\
\hline 19980812.03831870 & 021044.478 & +103015.64 & 19980816.12727870 & 021053.625 & +102959.13 \\
\hline 19980812.04135870 & 021044.485 & +103015.65 & 19980817.08377870 & 021054.797 & +102949.49 \\
\hline 19980812.04439870 & 021044.494 & +103015.63 & 19980817.09001870 & 021054.802 & +102949.46 \\
\hline 19980812.04743870 & 021044.503 & +103015.63 & 19980817.09780870 & 021054.806 & +102949.37 \\
\hline 19980812.05047870 & 021044.515 & +103015.68 & 19980817.10014870 & 021054.809 & +102949.39 \\
\hline 19980812.05350870 & 021044.520 & +103015.70 & 19980817.10249870 & 021054.810 & +102949.35 \\
\hline 19980812.05654870 & 021044.527 & +103015.71 & 19980817.10483870 & 021054.810 & +102949.32 \\
\hline 19980814.02856870 & 021049.828 & +103011.97 & 19980817.10718870 & 021054.810 & 49.30 \\
\hline 19980814.03405870 & 021049.818 & +103011.84 & 19980817.10952870 & & 949.33 \\
\hline 19980814.03913870 & & & 19980819.11656870 & & 924.02 \\
\hline 19980814.0421 & 0210 & +10 & 19980819.12047870 & 021055.935 & 923.99 \\
\hline 19980814.04520870 & 0210 & +103011.82 & 19980819.12399870 & 021055.932 & +102923.95 \\
\hline 19980814.04825870 & 0210 & 11.82 & 19980819.12770870 & 021055.932 & +102923.88 \\
\hline 19980814.05128870 & 021049.855 & +103011.83 & 19980819.13074870 & 021055.933 & +102923.81 \\
\hline 19980814.06712870 & 021049.888 & +103011.73 & 19980819.13378870 & 021055.933 & +102923.79 \\
\hline 19980814.07044870 & 021049.891 & +103011.79 & 19980819.13738870 & 021055.928 & +102923.72 \\
\hline 19980814.08282870 & 021049.917 & +103011.75 & 19980819.13973870 & 021055.931 & +102923.71 \\
\hline 19980814.08591870 & 021049.921 & +103011.72 & 19980819.14207870 & 021055.929 & +102923.66 \\
\hline 19980815.02335870 & 021051.863 & +103007.08 & 19980819.14441870 & 021055.928 & +102923.60 \\
\hline 19980815.03020870 & 021051.872 & +103007.07 & 19980819.14675870 & 021055.928 & +102923.59 \\
\hline 19980815.03495870 & 021051.881 & +103007.05 & 19980820.13088870 & 021055.865 & +102907.90 \\
\hline 19980815.03800870 & 021051.885 & +103007.01 & 19980820.13844870 & 021055.861 & +102907.71 \\
\hline 19980815.04103870 & 021051.889 & +103007.04 & 19980820.14452870 & 021055.857 & +102907.60 \\
\hline 19980815.04407870 & 021051.896 & +103006.97 & 19980820.14758870 & 021055.856 & +102907.54 \\
\hline 19980815.04711870 & 021051.895 & +103006.93 & 19980820.15061870 & 021055.856 & +102907.49 \\
\hline 19980815.05015870 & 021051.903 & +103006.91 & 19980820.15366870 & 021055.851 & +102907.44 \\
\hline 19980815.05319870 & 021051.910 & +103006.91 & 19980820.15670870 & 021055.849 & +102907.39 \\
\hline 19980815.05622870 & 021051.911 & +103006.85 & 19980820.15973870 & 021055.849 & +102907.33 \\
\hline 19980815.06618870 & 021051.931 & +103006.86 & 19980821.10810870 & 021055.403 & +102850.19 \\
\hline 19980815.06922870 & 021051.935 & +103006.85 & 19980821.11640870 & 021055.396 & +102850.03 \\
\hline 19980816.08740870 & 021053.580 & +102959.37 & 19980821.12019870 & 021055.391 & +102849.95 \\
\hline 19980816.09294870 & 021053.586 & +102959.33 & 19980821.12253870 & 021055.389 & +102849.90 \\
\hline 19980816.09620870 & 021053.594 & +102959.30 & 19980821.12487870 & 021055.387 & +102849.86 \\
\hline 19980816.09948870 & 021053.592 & +102959.19 & 19980821.12721870 & 021055.385 & +102849.82 \\
\hline 19980816.10274870 & 021053.600 & +102959.31 & 19980821.12954870 & 021055.382 & +102849.77 \\
\hline
\end{tabular}


Table 4. continued.

\begin{tabular}{|c|c|c|c|c|c|}
\hline $\begin{array}{c}\text { Date of Observation } \\
\text { Y M D UTC }\end{array}$ & $\begin{array}{l}\text { Right ascension } \\
\text { h m s.sss }\end{array}$ & $\begin{array}{l}\text { Declination } \\
\text { d m s.ss }\end{array}$ & $\begin{array}{l}\text { Date of Observation } \\
\text { Y M D UTC }\end{array}$ & $\begin{array}{c}\text { Right ascension } \\
\text { h m s.sss }\end{array}$ & $\begin{array}{l}\text { Declination } \\
\mathrm{d} \text { m s.ss }\end{array}$ \\
\hline 19980821.13189870 & 021055.381 & +102849.73 & 19981214.89155870 & 014617.603 & +081154.29 \\
\hline 19980821.13422870 & 021055.376 & +102849.64 & 19981214.89766870 & 014617.565 & +081154.03 \\
\hline 19980821.13657870 & 021055.376 & +102849.63 & 19981216.85578870 & 014604.550 & +081110.40 \\
\hline 19980821.13891870 & 021055.372 & +102849.60 & 19981216.85991870 & 014604.523 & +081110.29 \\
\hline 19980909.10898870 & 020929.345 & +10 16 39.19 & 19981216.86317870 & 014604.500 & +081110.21 \\
\hline 19980909.11254870 & 020929.316 & +10 1638.97 & 19981216.86644870 & 014604.481 & +081110.16 \\
\hline 19980909.11607870 & 020929.288 & +10 16 38.77 & 19981216.86970870 & 014604.458 & +081110.10 \\
\hline 19980909.11971870 & 020929.253 & +10 16 38.54 & 19981216.87297870 & 014604.436 & +08 1110.03 \\
\hline 19980909.12353870 & 020929.220 & +10 16 38.31 & 19981216.87624870 & 014604.415 & +08 1109.96 \\
\hline 19980910.11285870 & 020920.897 & +101541.36 & 19981217.87059870 & 014558.367 & +081051.59 \\
\hline 19980910.11857870 & 020920.846 & +101541.05 & 19981217.87550870 & 014558.337 & +081051.51 \\
\hline 19980910.12206870 & 020920.814 & +101540.85 & 19981217.87714870 & 014558.327 & +081051.51 \\
\hline 19980910.12557870 & 020920.782 & +101540.66 & 19981217.87879870 & 014558.315 & +081051.43 \\
\hline 19980910.12906870 & 020920.750 & +101540.43 & 19981217.88044870 & 014558.307 & +081051.41 \\
\hline 19981211.94689870 & 014639.919 & +081315.13 & 19981217.88209870 & 014558.296 & +081051.36 \\
\hline 19981211.95434870 & 014639.851 & +08 1314.83 & 19981217.88374870 & 014558.283 & +081051.32 \\
\hline 19981211.95877870 & 014639.820 & +081314.73 & 19981217.88539870 & 014558.273 & +081051.28 \\
\hline 19981211.96320870 & 014639.783 & +081314.61 & 19981217.88704870 & 014558.265 & +08 1051.28 \\
\hline 19981211.97058870 & 014639.722 & +08 1314.38 & 19981217.88868870 & 014558.255 & +081051.23 \\
\hline 19981211.97269870 & 014639.703 & +081314.25 & 19981217.89033870 & 014558.243 & +081051.19 \\
\hline 19981211.97480870 & 014639.685 & +081314.18 & 19991009.15478713 & 025520.072 & +135857.97 \\
\hline 19981212.88696870 & 014632.456 & +081247.20 & 19991009.15966713 & 025520.003 & +135857.62 \\
\hline 19981212.89320870 & 014632.405 & +081246.91 & 19991009.16518713 & 025519.933 & +135857.32 \\
\hline 19981212.89973870 & 014632.361 & +081246.75 & 19991009.16956713 & 025519.872 & +135857.03 \\
\hline 19981212.90300870 & 014632.327 & +081246.64 & 19991009.17315713 & 025519.823 & +135856.82 \\
\hline 19981212.90627870 & 014632.304 & +08 1246.57 & 19991009.17649713 & 025519.776 & +135856.58 \\
\hline 19981213.97741870 & 014624.166 & +08 1216.87 & 19991009.17975713 & 025519.732 & +135856.42 \\
\hline 19981213.98181870 & 014624.123 & +08 1216.93 & 19991011.15798713 & 025452.216 & +135646.88 \\
\hline 19981213.99001870 & 014624.061 & +08 1216.70 & 19991011.16180713 & 025452.160 & +135646.65 \\
\hline 19981213.99386870 & 014624.028 & +081216.56 & 19991011.16776713 & 025452.082 & +135646.50 \\
\hline 19981213.99770870 & 014624.003 & +08 1216.47 & 19991011.17071713 & 025452.039 & +135646.29 \\
\hline 19981214.82275870 & 014618.111 & +08 1155.83 & 19991011.17308713 & 025452.006 & +135646.13 \\
\hline 19981214.82700870 & 014618.078 & +08 1155.90 & 19991011.17550713 & 025451.970 & +135645.98 \\
\hline 19981214.83105870 & 014618.052 & +08 1155.67 & 19991011.17796713 & 025451.936 & +135645.82 \\
\hline 19981214.83632870 & 014618.010 & +08 1155.53 & 19991011.18044713 & 025451.899 & +135645.67 \\
\hline 19981214.84230870 & 014617.966 & +081155.35 & 19991011.18281713 & 025451.866 & +135645.49 \\
\hline 19981214.84745870 & 014617.927 & +08 1155.29 & 19991111.05207713 & 024621.072 & +131927.06 \\
\hline 19981214.85195870 & 014617.895 & +08 1155.14 & 19991111.05577713 & 024621.011 & +131926.84 \\
\hline 19981214.85639870 & 014617.863 & +08 1155.06 & 19991111.05696713 & 024620.987 & +131926.81 \\
\hline \multirow[t]{4}{*}{19981214.88545870} & 014617.657 & +081154.54 & 19991111.05814713 & 024620.970 & +131926.70 \\
\hline & & & 19991111.05932713 & 024620.949 & +131926.66 \\
\hline & & & 19991111.06050713 & 024620.927 & +131926.51 \\
\hline & & & 19991111.06050713 & 024620.927 & +131926.51 \\
\hline
\end{tabular}




\section{Conclusion}

Using several years of observations, we have increased the density of the set of astrometric observations of Phoebe. With optical observations of radio extragalactic sources, a zonal error of the USNO A2.0 catalogue was detected (Fienga \& Andrei 2002). The Phoebe observations were corrected for this zonal error. Furthemore, one can notice that the size of the residuals of the new observations against the current ephemerides seems to indicate that the ephemerides probably need very little further adjustment. This work shows that it is possible to get valuable observations for the improvement of Phoebe's orbit using a quite small telescope $(1.2 \mathrm{~m})$. However, new observations are necessary to cover a sufficient number of orbits. We look forward to new observations and new observers of the Saturn satellite, Phoebe.

Acknowledgements. These observations have been made possible thanks to the CNRS (Centre National de la Recherche Scientifique), the Institut des sciences de l'Univers/CNES through the Programme National de Planétologie and the Institut de mécanique céleste/Bureau des Longitudes.

We also wish to thank the staff of the Observatoire de HauteProvence (OHP) for their efforts and their help during the campaign.

The Guide Star Catalogue-II is a joint project of the Space Telescope Science Institute and the Osservatorio Astronomico di Torino. Space Telescope Science Institute is operated by the Association of Universities for Research in Astronomy, for the National Aeronautics and Space Administration under contract NASA5-26555. The participation of the Osservatorio Astronomico di Torino is supported by the Italian Council for Research in Astronomy. Additional support is provided by European Southern Observatory, Space Telescope European Coordinating Facility, the International GEMINI project and the European Space Agency Astrophysics Division.

\section{References}

Arlot, Borsenberger, A., Fienga, A., \& Baron, N. 2002, A\&A, in preparation

Assafin, M., Andrei, A. H., Vieira Martins, R., et al. 2001, ApJ, 552, 380

Banse, K., Grosbol, P. J., \& Baade, D. 1991, Astronomical Data Analysis Software and Systems I, PASP Conf. Ser., 25, 120

Bertin, E. 1998, http://www . eso.org/science/eis

Berthier, J., \& Fienga, A. 1999, Proc. of Journées Scientifiques de l'Institut de Mécanique Céleste, Paris

Berthier, J., Fienga, A., \& Descamps, P. 2000, Proc. of Journées Scientifiques de l'Institut de Mécanique Céleste, June 7-9, Paris

Bonnet-Bidaud, J. M., Colas, F., \& Lecacheux, J. 2000, A\&A, 360, 991

Charlot, P. 1990, AJ, 99, 1309

Fey, A. L., \& Charlot, P. 1997, ApJS, 111, 95

Fienga, A. 1998, A\&A, 335, 1111

Fienga, A. 1999, Ph.D. Thesis, Observatoire de Paris

Fienga, A., \& Andrei, A. H. 2002, A\&A, submitted

Fienga, A., Colas, F., Lecacheux, J., \& Berthier, J. 2002, A\&A, in preparation

Jacobson, R.A. 1998, A\&AS, 128, 7

Morrison, J. E., Roeser, S., McLean, B., Bucciarelli, B., \& Lasker, B. 1999, VizieR Online Data Catalog, 1254

Mink, D. J. 1995, Proc. of Fifth Annual Conference on Astronomical Data Analysis Software and Systems, 101, 96

Monet, D., Bird, A., Canzian, B., et al. 1998, The USNO-A2.0 Catalogue, (U.S. Naval Observatory, Washington DC)

Stone, R. C., \& Harris, F. H. 2000, AJ, 119, 1985

Stone, R. C. 2000, AJ, 120, 2124

STScI 2001, http://www-gsss. stsci . edu/gsc/gsc2/ GSC2home.htm

Urban, S. E., Corbin, T. E., \& Wycoff, G. L. 1998, AJ, 115, 2161

Veiga, C. H., Vieira Martins, R., \& Andrei, A. H. 2000, A\&AS, 142, 81

Zacharias, N., Zacharias, M. I., Hall, D. M., et al. 1999, AJ, 118, 2511 